Original Research Paper

\title{
An Improved Positivity Preserving Odd Degree-N Said-Ball Boundary Curves on Rectangular Grid Using Partial Differential Equation
}

\author{
${ }^{1}$ Ahmed Kherd, ${ }^{2}$ Azizan Saaban and ${ }^{2}$ Noraziah Haji Man \\ ${ }^{I}$ Faculty of Computer Scince and Engineering, AL-Ahgaff Universty Mukalla, Hadramout, Yemen \\ ${ }^{2}$ UUM College of Arts and Sciences, Universty Utara Malaysia, Sintok, Kedah, Malaysia
}

\author{
Article history \\ Received: 18-09-2014 \\ Revised: 17-02-2016 \\ Accepted: 24-02-2016 \\ Corresoonding Author: \\ Ahmed Kherd \\ Faculty of Computer Scince \\ and Engineering, AL-Ahgaff \\ Universty Mukalla, Hadramout, \\ Yemen \\ E-mail: as_kherd@hotmail.com
}

\begin{abstract}
This paper discusses the sufficient conditions for positivity preserving odd degree- $n$ Said-Ball boundary curves defined on a rectangular grid. We derive a sufficient condition on boundary curves of rectangular Said-Ball patches where the lower bound ordinates are adjusted independently. To construct the boundary curves for each rectangular patch, the Said-Ball polynomial solution of fourth order PDE will be considered where its coefficients can be calculated using edge Said-Ball ordinates which fulfill the positivity preserving conditions. Graphical examples are presented using well-known test functions.
\end{abstract}

Keywords: Positivity, Rectangular Said-Ball Patches, Partial Differential Equation

\section{Introduction}

In this study we will discuss an explicit polynomial solution method for surface generation which is characterized by some positivity preserving Said-Ball boundary curves whereby the resulting surface conforms to a linear elliptic Partial Differential Equation (PDE). There are many areas of science and engineering which are modeled using fourth order linear elliptic PDEs, for example, in material sciences (stress/strain in physical structures), biology (fluid dynamics inside lungs), image processing (enhancement of noisy images) and computer aided geometric design (surface design), as discussed in (Arnal et al., 2011). The PDE methods solve a boundary value problem subject to four boundary curves condition which are widely discussed as seen in (Bloor and Wilson, 1990; Monterde and Ugail, 2004; 2006; Arnal et al., 2011. All these previous works discussed the construction of surface interpolation on single rectangular patch subject to four boundary curves while our proposed method will consider the construction of smooth surface defined on uniformly distributed scattered data using the combination of rectangular patches.

This study is an extension of the previous works in Saaban et al. (2006) and in Kherd et al. (2014) which described the sufficient condition on boundary curves of bicubic rectangular Bézier patches and bicubicrectangular Said-Ball patches respectively where the lower bound of edge ordinates are adjusted independently. The construction of quintic boundary curves for each rectangular patch involves considering the use of Said-Ball polynomial solution of fourth order PDE as discussed in Monterde and Ugail (2006). In order to derive a relation between Said-Ball boundary and polynomial coefficients of Said-Ball patch of degree $m$ by $n$, we write Said-Ball patch as Bézier patch of degree $m$ by $n$ as in Equation 7 .

The coefficients of the polynomial form are calculated from the edge Said-Ball ordinates of quintic rectangular patch which fulfill the positivity preserving conditions. The problem we are discussing here is that for a given four positivity preserving boundary curves in a unit square $0 \leq u, v \leq 1$, we will generate a parametric surface patch $X(u, v)$ in $R^{3}$ that smoothly interpolates these boundary curves and then the interpolating surface of a given uniform positive scattered data will be constructed. We will describe the generalized form of sufficient conditions for the positivity preserving odd degree- $n$ Said-Ball boundary curves for a given scattered data defined on a rectangular grid. 


\section{Said-Ball Surface}

The degree $m$ by $n$ Said-Ball patch $X$ is defined as:

$$
X(u, v)=\sum_{i, j=0}^{m, n} S_{i}^{m}(u) S_{j}^{n}(v) v_{i j}
$$

where, $v_{i j}$ are the control points and $S_{i}^{m}(u), S_{j}^{n}(v)$ are SaidBall basis functions with:

$$
S_{i}^{n}(u)=\left\{\begin{array}{l}
\left(\begin{array}{c}
\frac{n-1}{2}+i \\
i
\end{array}\right) u^{i}(1-u)^{\frac{n+1}{2}} \text { if } \quad 0 \leq i \leq \frac{n-1}{2} \\
\left(\begin{array}{c}
\frac{n-1}{2}+n-i \\
n-i
\end{array}\right) u^{\frac{n+1}{2}}(1-u)^{n-i}, \text { if } \quad \frac{n+1}{2} \leq i \leq n
\end{array}\right.
$$

When $n$ is odd and:

$$
S_{i}^{n}=\left\{\begin{array}{l}
\left(\begin{array}{l}
\frac{n}{2}+i \\
i
\end{array}\right) u^{i}(1-u)^{\frac{n}{2}+1}, \text { if } 0 \leq i \leq \frac{n}{2}-1 \\
\left(\begin{array}{l}
\frac{n}{2} \\
\frac{n}{2}
\end{array}\right) u^{\frac{n}{2}}(1-u)^{\frac{n}{2}}, \text { if } \quad i=\frac{n}{2} \\
\left(\begin{array}{l}
\frac{n}{2}+n-i \\
n-i
\end{array}\right) u^{\frac{n}{2}+1}(1-u)^{n-i}, \quad \text { if } \frac{n}{2}+1 \leq i \leq n
\end{array}\right.
$$

When $n$ is even, as in Aphirukmatakun and Dejdumrong, 2007; Hu et al., 1996).

Now we convert Equation1 into Bèzier Surface of degree $m$ by $n$.

The formula for converting Said-Ball Curve to Bézier curve was given by $\mathrm{Hu}$ et al. (1996) as:

$$
\left[\begin{array}{c}
S_{0}^{n}(u) \\
S_{1}^{n}(u) \\
\vdots \\
S_{i}^{n}(u) \\
\vdots \\
S_{n}^{n}(u)
\end{array}\right]=\left[\begin{array}{ccccc}
c_{00} & c_{01} & \ldots & \ldots & c_{0 n} \\
c_{10} & c_{11} & \ddots & \ddots & c_{1 n} \\
\vdots & \vdots & \ddots & \ddots & \vdots \\
c_{i 0} & c_{i 1} & \ddots & \ddots & c_{i n} \\
\vdots & \vdots & \ddots & \ddots & \vdots \\
c_{n 0} & c_{n 1} & \ldots & \ldots & c_{n n}
\end{array}\right]\left[\begin{array}{c}
B_{0}^{n}(u) \\
B_{1}^{n}(u) \\
\vdots \\
B_{i}^{n}(u) \\
\vdots \\
B_{n}^{n}(u)
\end{array}\right]
$$

where, $S_{i}^{n}(u)$ and $B_{i}^{n}(u)$, for $i \in\{0,1, \ldots, n\}$ is the SaidBall basis functions and Bernstein basis functions respectively and $C$ is the convert matrix given by:

$$
\left\{\begin{array}{l}
\frac{\left(\begin{array}{c}
\frac{n}{2}+i \\
i
\end{array}\right)\left(\begin{array}{c}
\frac{n}{2}-1-i \\
j-i
\end{array}\right)}{\left(\begin{array}{c}
n \\
j
\end{array}\right)}, i \leq j \leq \frac{n}{2}-1 \\
\frac{\left(\begin{array}{c}
\frac{3}{2} n-i \\
n-i
\end{array}\right)\left(\begin{array}{c}
i-1-\frac{n}{2} \\
i-j
\end{array}\right)}{\left(\begin{array}{l}
n \\
j
\end{array}\right)}, i \geq j \geq \frac{n}{2}+1 \\
1, \quad i=j=\frac{n}{2} 0, \quad \text { otherwise }
\end{array}, i\right.
$$

When $n$ is even and:

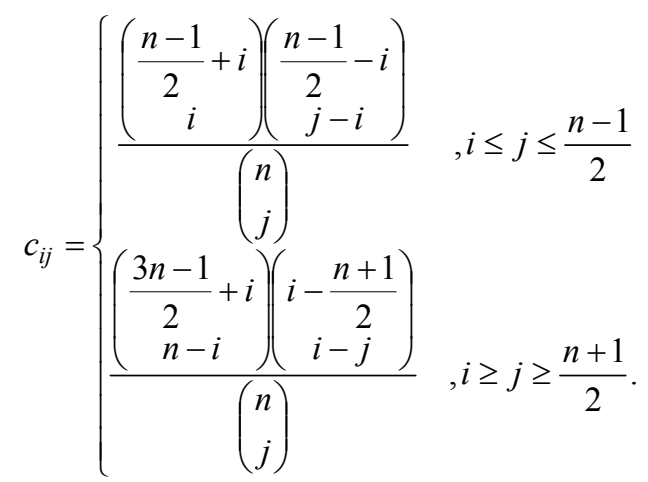

When $n$ is odd.

The Said-Ball surface of degree $m \times n$ in Equation1 can be written in matrix form as:

$X(u, v)=S_{u} V S_{v}$

where:

$$
\begin{aligned}
S_{u} & =\left[\begin{array}{llll}
S_{0}^{m}(u) & S_{1}^{m}(u) & \ldots & S_{m}^{m}(u)
\end{array}\right] \\
S_{v} & =\left[\begin{array}{llll}
S_{0}^{n}(v) & S_{1}^{n}(v) & \ldots & S_{n}^{n}(v)
\end{array}\right]^{t} \\
V & =\left[\begin{array}{cccc}
v_{00} & v_{01} & \ldots & v_{0 n} \\
v_{10} & v_{11} & \ldots & v_{1 n} \\
\vdots & \vdots & \ddots & \vdots \\
v_{m 0} & v_{m 1} & \ldots & v_{m n}
\end{array}\right]
\end{aligned}
$$

and $v_{i j}$, for $i \in\{0,1, \ldots, m\}, j \in\{0,1, \ldots, n\}$ are the control points of Said-Ball surface.

By using Equation 4 in 5, we get: 


$$
\begin{aligned}
& X(u, v)=\left(C B_{u}\right)^{t} V\left(D B_{v}\right) \\
& =\left(B_{u}^{t} C^{t}\right) V\left(D B_{v}\right) \\
& =B_{u}^{t}\left(C^{t} V D\right) B_{v} \\
& =B_{u}^{t} P B_{v}
\end{aligned}
$$

where, $P=C^{t} V D$ and $C$ and $D$ are convert matrices of order $m$ and $n$ respectively.

Now we rewrite Equation6 as:

$$
X(u, v)=\sum_{i, j=0}^{m, n} B_{i}^{m}(u) B_{j}^{n}(v) P_{i j}
$$

Which is the Bézier surface of degree $m \times n$.

\section{Sufficient Condition for Positivity Preserving Odd Degree-N Said-Ball Curves}

The sufficient conditions for positivity preserving quintic curves is given in the following proposition.

\section{Proposition 1.}

Consider the odd degree- $n(n \geq 3)$ Said-Ball ponomial curve:

$$
r(x)=\sum_{i=0}^{n} S_{i}^{n}(x) v_{i}
$$

where, $n$ is odd, $v_{i}$ are Said-Ball control points and $S_{i}^{n}(x), \forall 0 \leq x \leq 1$.

If $v_{0}, v_{n}>0, M=\max \left(v_{0}, v_{n}\right), N=\min \left(v_{0}, v_{n}\right)$ and $v_{i} \geq-t_{0}=-\frac{1}{s_{0}}, i \neq 0, i \neq n$ where $s_{0}$ in $\left[\frac{2^{\frac{n-1}{2}}-1}{M}, \frac{2^{\frac{n-1}{2}}-1}{N}\right]$ is the unique solution of $G(s)=1$ with $G(s)=\frac{1}{\left(s v_{0}+1\right)^{\frac{2}{n-1}}}+\frac{1}{\left(s v_{n}+1\right)^{\frac{2}{n-1}}}$, then $\mathrm{r}(\mathrm{x}) \geq 0, \forall x \in[0,1]$.

\section{Proof}

Let all Said-Ball ordinates of odd degree-n Said-Ball polynomial curve except for $v_{0}, v_{n}$ are equal to $-t(t>0)$ Then, Equation 8 can be written as:

$$
r(x)=v_{0}(1-x)^{\frac{n+1}{2}}+\sum_{i=1}^{n-1}(-t) S_{i}^{n}(x)+v_{n} x^{\frac{n+1}{2}}
$$

That is equivalent to:

$$
r(x)=v_{0}(1-x)^{\frac{n+1}{2}}-t \sum_{i=1}^{n-1} S_{i}^{n}(x)+v_{n} x^{\frac{n+1}{2}} .
$$

Recall that:

$$
\sum_{i=0}^{n} S_{i}^{n}(x)=(1-x)^{\frac{n+1}{2}}+\sum_{i=1}^{n-1} S_{i}^{n}(x)+x^{\frac{n+1}{2}}=1
$$

That is equivalent to:

$$
\sum_{i=1}^{n-1} S_{i}^{n}(x)+=1-(1-x)^{\frac{n+1}{2}}-x^{\frac{n+1}{2}}
$$

Using Equation 10 in 9, we get:

$$
r(x)=\left(v_{0}+t\right)(1-x)^{\frac{n+1}{2}}+\left(v_{n}+t\right) x^{\frac{n+1}{2}}-t
$$

Assume that $t$ is fixed. Then, the first and second derivatives of $r$ with respect to $x$ are:

$$
r^{\prime}(x)=\frac{n+1}{2}\left(-\left(v_{0}+t\right)(1-x)^{\frac{n-1}{2}}+\left(v_{n}+t\right) x^{\frac{n-1}{2}}\right)
$$

and:

$$
r^{\prime \prime}(x)=\frac{(n+1)(n-1)}{4}\left(\left(v_{0}+t\right)(1-x)^{\frac{n-3}{2}}+\left(v_{n}+t\right) x^{\frac{n-3}{2}}\right)
$$

Setting $r^{\prime}(x)=0$ gives:

$$
\frac{(1-x)^{\frac{n-1}{2}}}{x^{\frac{n-1}{2}}}=\frac{v_{n}+t}{v_{0}+t}, x \neq 0 \frac{1-x}{x}=\left(\frac{v_{n}+t}{v_{0}+t}\right)^{\frac{2}{n-1}}, x \neq 0
$$

Thus, we get:

$$
x=\frac{\left(v_{0}+t\right)^{\frac{2}{n-1}}}{\left(v_{0}+t\right)^{\frac{2}{n-1}}+\left(v_{n}+t\right)^{\frac{2}{n-1}}}
$$

It follows that $v_{0}>0$ and $v_{n}>0,0<x \leq 1$, which implies that $r "(x)>0$. Then the minimum value of $r$ occurs when $x=\frac{\left(v_{0}+t\right)^{\frac{2}{n-1}}}{\left(v_{0}+t\right)^{\frac{2}{n-1}}+\left(v_{n}+t\right)^{\frac{2}{n-1}}}$ that is:

$$
r_{\min }(x)=\frac{\left(v_{0}+t\right)\left(v_{n}+t\right)}{\left(\left(v_{0}+t\right)^{\frac{2}{n-1}}+\left(v_{0}+t\right)^{\frac{2}{n-1}}\right)^{\frac{n-1}{2}}}-t
$$

Observe that if $t=0, r_{\min }(x)>0$ preserves the positivity for all $0 \leq x \leq 1$. Rewriting Equation 12, we obtain:

$$
r_{\min }(x)=\frac{1}{\left(\frac{1}{\left(v_{0}+t\right)^{\frac{2}{n-1}}}+\frac{1}{\left(v_{0}+t\right)^{\frac{2}{n-1}}}\right)^{\frac{n-1}{2}}}-t
$$


Which is equivalent to:

$$
r_{\min }(x)=\frac{t}{\left(\left(\frac{v_{0}}{t}+1\right)^{\frac{2}{n-1}}+\left(\frac{v_{n}}{t}+1\right)^{\frac{2}{n-1}}\right)^{\frac{n-1}{2}}}-t, t>0
$$

The Said-Ball polynomial curve $r(x)$ is positive for all $x$ if $r_{\min }(x)>0$. Thus the lower bound of $t=t_{0}$ occurs when $r_{\text {min }}=0$ and this can be achieved by setting the denominator of Equation 13 equal to 1. Therefore:

$$
\left(\frac{v_{0}}{t}+1\right)^{\frac{2}{n-1}}+\left(\frac{v_{n}}{t}+1\right)^{\frac{2}{n-1}}=1
$$

Let $s=\frac{1}{t}, t \neq 0 \quad$ and $\quad G(s)=\frac{1}{\left(s v_{0}+1\right)^{\frac{2}{n-1}}}+\frac{1}{\left(s v_{n}+1\right)^{\frac{2}{n-1}}}$ then Equation 14 becomes:

$$
G(s)=1, s \geq 0
$$

Since $v_{0}>0$ and $v_{n}>0$ then $s_{0}$ is the solution of Equation 14 and the value of $t_{0}$ is equal to $\frac{1}{s_{0}}$ where $s_{0}>$ 0. Thus $r(x) \geq 0$, for all $x \in[0,1]$ if $v_{i} \geq-t{ }_{0}=\frac{1}{s_{0}}, i \neq 0, i \neq n$ where $s_{0}$ is the unique solution of $G(s)=1$ with $G(s)=\frac{1}{\left(s v_{0}+1\right)^{\frac{2}{n-1}}}+\frac{1}{\left(s v_{n}+1\right)^{\frac{2}{n-1}}}$.

We shall prove that there exists $\frac{2^{\frac{n-1}{2}}-1}{M} \leq s_{0} \leq \frac{2^{\frac{n-1}{2}}-1}{N}$ for $G(s)=1$ where $M=\max \left(v_{0}, v_{n}\right)$ and $N=\min \left(v_{0}, v_{n}\right)$.

Since:

$$
G^{\prime}(s)=-\frac{2}{n-1}\left(\frac{v_{0}}{\left(s v_{0}+1\right)^{\frac{n+1}{n-1}}}+\frac{v_{n}}{\left(s v_{n}+1\right)^{\frac{n+1}{n-1}}}\right) \leq 0
$$

and:

$$
G^{\prime \prime}(s)=\frac{2(n+1)}{(n-1)^{2}}\left(\frac{\left(v_{0}\right)^{2}}{\left(s v_{0}+1\right)^{\frac{2 n}{n-1}}}+\frac{\left(v_{n}\right)^{2}}{\left(s v_{n}+1\right)^{\frac{2 n}{n-1}}}\right) \geq 0
$$

Then $G(s)$ is monotone decreasing and convex. Since $\lim G(s)=0$ and $G(0)=2$ and by the monotonicity of $G(s)$, then there exist $s$ subject to $G(0)=1$. Now we need to determine the range of $s$.

$$
\text { If } v_{0}=v_{n}=\max \left(v_{0}, v_{n}\right)=M \text {, then } G(s) \geq \frac{2}{(M s+1)^{\frac{2}{n-1}}}
$$

and if $v_{0}=v_{n}=\min \left(v_{0}, \quad v_{n}\right)=N$, then $G(s) \leq \frac{2}{(N s+1)^{\frac{2}{n-1}}}$ since $\frac{2}{(N s+1)^{\frac{2}{n-1}}}$ and $\frac{2}{(M s+1)^{\frac{2}{n-1}}}$ are also monotone decreasing and convex, then there exists $s_{1}$ and $s_{2}$ such that $\frac{2}{\left(M s_{1}+1\right)^{\frac{2}{n-1}}}=1$, $s_{1}=\frac{2^{\frac{n-1}{2}}-1}{M}$ and $s_{2}=\frac{2^{\frac{n-1}{2}}-1}{N}$.

Thus if $G(s)=1$, then $s_{1} \leq s_{0} \leq s_{2}$. Figure 1 shows the form of $G(s), s \geq 0$ with the relative location of $s_{1}=\frac{2^{\frac{n-1}{2}}-1}{M}, s_{2}=\frac{2^{\frac{n-1}{2}}-1}{N}$ and $s=s_{0}$.

To obtain the value of $s_{0}$ forgiven values of $v_{0}$ and $v_{n}$, we need to calculate the root of Equation 15 that will give a lower bound on the remaining Said-Ball ordinates, that is, $t_{0}=1 / s_{0}$. We can use a simple iterative scheme which must ensure one-sided convergence, where $s_{0}$ is approached from above. The convexity of $G(s)$ means that this can be achieved using the false-positions method of Conte and de Boor (1992). An initial estimate for the root will be the value of $s_{0}$ for which the line joining $s_{1}$ and $s_{2}$ has the value 1 as shown in Fig. 1 .

\section{Sufficient Condition for Positivity Preserving Odd Degree-N Siad-Ball Boundary Curves Defined On Rectangular Grid}

Consider a rectangle $R$ with vertices $V_{1}, V_{2}, V_{3}$ and $V_{4}$ and we denote the edges along $V_{1} V_{2}, V_{4} V_{3}, V_{1} V_{4}$ and $V_{2} V_{3}$ by $e_{1}, e_{2}, e_{3}$ and $e_{4}$ respectively. Rectangular Said-Ball patch $P$ of degree $m$ by non $R$ is defined as:

$$
P(u, v)=\sum_{i, j=0}^{m, n} S_{i}^{m}(u) S_{j}^{n}(v) v_{i j}
$$

where, $v_{i j}$ are the control points and $S_{i}^{m}(u), S_{j}^{n}(v)$ are SaidBall basis functions. The four boundary curves along the edges $e_{1}, e_{2}, e_{3}$ and $e_{4}$ are given by $C_{1}(u), C_{2}(u), C_{3}(v)$ and $C_{4}(v)$ respectively with:

$$
\begin{aligned}
& C_{1}(u)=P(u, 0)=\sum_{i=0}^{m} v_{i 0} S_{i}^{m}(u) \\
& C_{2}(u)=P(u, 1)=\sum_{i=0}^{m} v_{i n} S_{i}^{m}(u) \\
& C_{3}(v)=P(0, v)=\sum_{i=0}^{n} v_{0 i} S_{i}^{n}(v) \\
& C_{4}(v)=P(1, v)=\sum_{i=0}^{n} v_{m i} S_{i}^{n}(v)
\end{aligned}
$$




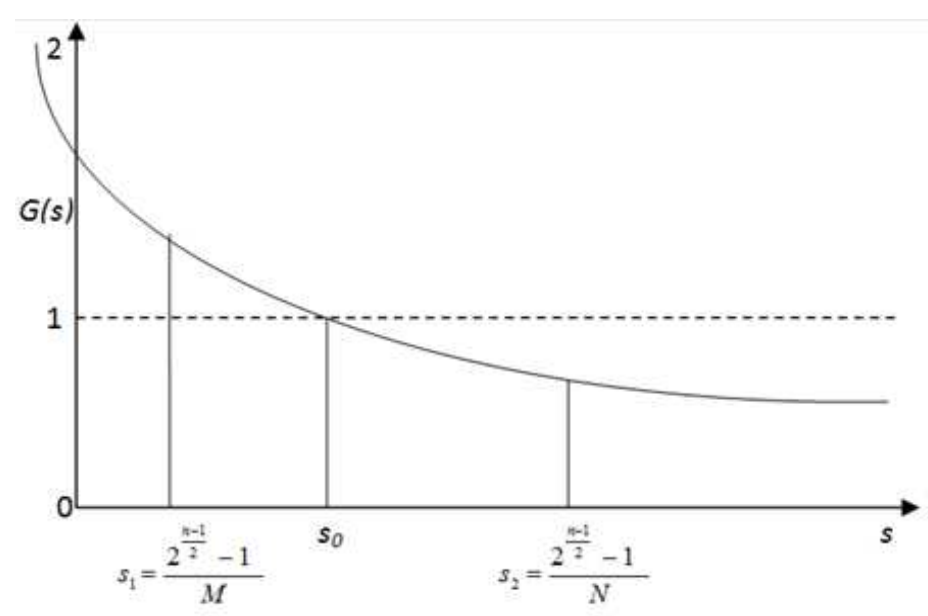

Fig. 1. Function $G(s)$ with $s_{0}$ for odd degree-n Said-Ball polynomial curve

Now, we shall proceed to determine the lower bound on the edges Said-Ball ordinates which ensure the positivity of boundary curves of odd degree- $n$ rectangular Said-Ball patch $P$. We shall derive the sufficient conditions for positivity preserving boundary curves for a given positive Said-Ball ordinates at vertices of rectangle $R$ that is $v_{00}, v_{m 0}, v_{0 n}, v_{m n}>0$. In order to ensure the positivity of boundary curves $P(u, 0), P(u, 1)$, $P(0, v)$ and $P(1, v)$, we set $v_{i 0} \geq-t_{1}, v_{i n} \geq-t_{2}, v_{0 j} \geq-t_{3}$ and $v_{m j} \geq-t_{4}$ for $1 \leq i \leq m-1,1 \leq j \leq n-1$ where $t_{1}, t_{2}, t_{3}, t_{4},>0$ can be obtained from Equation 14 with $v_{0}=v_{00}, v_{m}=v_{m 0}$ along edge $e_{1}, v_{0}=v_{0 n}, v_{m}=v_{m n}$ along edge $e_{2}, v_{0}=v_{00}, v_{m}$ $=v_{0 n}$ along edge $e_{3}$ and $v_{0}=v_{m 0}, v_{m}=v_{m n}$ along edge $e_{4}$. Since $C_{1}(u), C_{2}(u), C_{3}(v)$ and $C_{4}(v)$ are the odd degree-n Said-Ball polynomial curve, we can use Proposition 1 to obtain the positivity preserving conditions for each boundary curve.

\section{Surface Interpolation Using Positivity Preserving Boundary Curves}

Rectangular Said-Ball patch $\mathrm{P}$ of degree $\mathrm{m}$ by non $\mathrm{R}$ as defined in Equation 16 is:

$$
P(u, v)=\sum_{i, j=0}^{m, n} S_{i}^{m}(u) S_{j}^{n}(v) v_{i j}, 0 \leq u, v \leq 1
$$

where, $v_{i j}$ are the control points and $S_{i}^{m}(u), S_{j}^{n}(v)$ are SaidBall basis functions. Using the first and second order partial derivatives of interpolating surface $S$ and the functional values at node $\left(x_{i}, y_{i}\right), \mathrm{i}=1,2,3,4$ the initial value of boundary Said-Ball ordinates $\mathrm{v}_{\mathrm{ij}}$ from Equation 14 which are $j_{v 0}, v_{j n}, v_{o j}, v_{n j}, j=1,2,3,4$ can be calculated.

The initial partial derivatives at the vertices of the rectangular patch were estimated by using forward, backward and central difference methods. In order to ensure the positivity of boundary curves, we need to adjust the partial derivatives at the vertices of the rectangle to fulfill the conditions $v_{j 0} \geq-t_{1}, v_{j n} \geq-t_{2}, v_{0 j} \geq-t_{3}$ and $v_{n j} \geq-$ $t_{4}$. The modification of these partial derivatives at vertex $V_{i}$, is achieved by multiplying each derivative at that vertex, by a scaling factor $0<a<1$ which is discussed in Saaban et al. (2007). In order to derive the relation between Said-Ball boundary and polynomial coefficients, Said-Ball patch of degree $m \times n$ can be written as Bézier patch of degree $m \times n$ by using Equation 7 as follows:

$P(u, v)=\sum_{i, j=0}^{m, n} B_{i}^{m}(u) B_{j}^{n}(v) P_{i j}, 0 \leq u, v \leq 1$

where, $P_{i j}$ are the Bézier control points and $B_{i}^{r}(u)=\frac{r !}{i !(r-i) !} u^{i}(1-u)^{(r-i)}$.

For the purpose of our numerical examples of uniform scattered data, we will consider fourth order linear elliptic PDE in the form of:

$A \frac{\partial^{4} X}{\partial u^{3} \partial v}+B \frac{\partial^{4} X}{\partial u^{2} \partial v^{2}}+C \frac{\partial^{4} X}{\partial u \partial v^{3}}=0$

Subject to given four line boundary conditions from a positivity preserving quintic Said-Ball curve $(n=5)$ defined by Equation 18 where $A, B$ and Care free parameters. We will compute Said-Ball polynomial solution of Equation 19 to be of the form of Equation 18. In order to generate positivity preserving of the boundary curves on each rectangle, we shall write Equation 18 in polynomial form $(n=5)$ :

$X(u, v)=\sum_{i, j=0}^{5} \frac{a_{i j}}{i ! j !} u^{i} v^{j}, 0 \leq u, v \leq 1$

Using similar approach of Monterde and Ugail (2006), the first and second rows (columns) of the 
coefficients in Equation 20 can be obtained by using the boundary control points of Said-Ball representation as in Equation 18 and is described as:

$$
X(u, v)=\sum_{i, j=0}^{5} \frac{a_{i j}}{i ! j !} u^{i} v^{j}=\sum_{i=0}^{5} \sum_{j=0}^{5} p_{i j} B_{i}^{5}(u) B_{j}^{5}(v)
$$

The relation between the first row of polynomial coefficients, $\left\{a_{0 j}\right\}_{j=0}^{5}$ with the boundary control points $\left\{p_{0 j}\right\}_{j=0}^{5}$ and first column coefficients $\left\{a_{j 0}\right\}_{j=0}^{5}$ with the boundary control points $\left\{p_{j 0}\right\}_{j=0}^{5}$ can be determined by taking $u=0$ and $v=0$ in Equation 21 respectively. Thus we have:

$$
a_{i 0}=i !\left(\sum_{k=0}^{i} E_{k, i-k}^{5} p_{k 0}\right), i=0, \ldots, 5
$$

and:

$$
a_{0 j}=j !\left(\sum_{k=0}^{j} E_{k, j-k}^{5} p_{0 k}\right), j=0, . ., 5
$$

where:

$$
\begin{gathered}
E_{i, j}^{5}=(-1)^{j}\left(\begin{array}{l}
5 \\
i
\end{array}\right)\left(\begin{array}{l}
5-i \\
j
\end{array}\right), \text { i.e. } \\
a_{i j}=0, \forall i+j>n+1 \\
a_{00}=v_{00}, a_{01}=-3\left(v_{00}-v_{01}\right) \\
a_{02}=6\left(v_{00}-3 v_{01}+2 v_{02}\right) \\
a_{03}=-6\left(v_{00}-9 v_{01}+18 v_{02}-6 v_{03}-3 v_{04}-v_{05}\right) \\
a_{04}=-72\left(v_{01}-6 v_{02}+4 v_{03}\right) \\
a_{05}=-720\left(v_{02}-v_{03}\right) \\
a_{10}=-3\left(v_{00}-v_{10}\right), a_{20}=6\left(v_{00}-3 v_{10}+2 v_{20}\right) \\
a_{30}=-6\left(v_{00}-9 v_{10}+18 v_{20}-6 v_{30}-3 v_{40}-v_{50}\right) \\
a_{40}=-72\left(v_{10}-6 v_{20}+4 v_{30}\right) \\
a_{50}=-720\left(v_{20}-v_{30}\right)
\end{gathered}
$$

where, $v_{i j}$ are control points of the Said-Ball surface of degree $m \times n$.

Next we shall calculate the second row $\left\{a_{5 j}\right\}_{j=1}^{5}$, the second column $\left\{p_{j 5}\right\}_{j=1}^{5}$ and the remaining coefficients of the polynomial coefficients in terms of the boundary control point of Said-Ball patch by using Equation 21 and by taking $u=1$ and $v=1$ in Equation 19 respectively. Thus, we obtain the following relations:

$$
\begin{aligned}
& a_{15}=-a_{05}-\left(v_{52}-v_{53}\right) \\
& a_{51}=-a_{50}-\left(v_{25}-v_{35}\right) \\
& a_{24}=\left(A^{3} a_{51}+C\left(B^{2}-A C\right) a_{15}\right) /\left(B\left(2 A C-B^{2}\right)\right) \\
& a_{42}=\left(C^{3} a_{15}+A\left(B^{2}-A C\right) a_{51}\right) /\left(B\left(2 A C-B^{2}\right)\right) \\
& a_{14}=-a_{04}-\frac{a_{24}}{2}-72\left(v_{51}-6 v_{52}+4 v_{53}+v_{54}\right) \\
& a_{41}=-a_{40}-\frac{a_{42}}{2}-72\left(v_{15}-6 v_{25}+4 v_{35}+v_{45}\right) \\
& a_{33}=\left(A^{2} a_{51}+C^{2} a_{15}\right) /\left(B^{2}-A C\right) \\
& a_{23}=\left(B C a_{14}-A^{2} a_{41}\right) /\left(B^{2}-A C\right) \\
& a_{32}=\left(C^{2} a_{14}-A B a_{41}\right) /\left(A C-B^{2}\right) \\
& a_{13}=-6\left(v_{50}-9 v_{51}+18 v_{52}-6 v_{53}-3 v_{54}-v_{55}\right)-a_{30} \\
& -\frac{a_{23}}{2}-\frac{a_{33}}{6}, \\
& a_{31}=-6\left(v_{05}-9 v_{15}+18 v_{25}-6 v_{35}-3 v_{45}-v_{55}\right)-a_{03} \\
& -\frac{a_{32}}{2}-\frac{a_{33}}{6} \\
& a_{22}=-\left(A a_{31}+C a_{13}\right) \\
& a_{12}=-a_{02}-\frac{a_{22}}{2}-\frac{a_{32}}{6}-\frac{a_{42}}{24}+6\left(v_{50}-3 v_{51}+2 v_{52}\right) \\
& a_{21}=-a_{20}-\frac{a_{22}}{2}-\frac{a_{23}}{6}-\frac{a_{24}}{24}+6\left(v_{05}-3 v_{15}+2 v_{25}\right) \\
& a_{11}=-\frac{a_{21}}{2}-\frac{a_{31}}{6}-\frac{a_{41}}{24}-\frac{a_{51}}{120}+3\left(v_{00}-v_{01}-v_{50}+v_{51}\right)
\end{aligned}
$$

where, $v_{i j}$ are control points of Said-Ball surface of degree $\mathrm{m} \times \mathrm{n}$ and $A, B$ and $C$ where $B \neq 0$ are free parameters.

\section{Graphical Examples}

To visualize our proposed method, we have chosen two datasets taken from well known test functions $f$ and $g$ where:

$$
f(x, y)=\left\{\begin{array}{l}
1.0, \text { if }(y-x) \geq 0 \\
2(y-x), \text { if } 0.5 \geq(y-x) \geq 0.0 \\
\cos \left(4 \pi \sqrt{(x-1.5)^{2}+(y-0.5)^{2}}+1\right) / 2 \\
\text { if }(x-1.5)^{2}+(y-0.5)^{2} \leq \frac{1}{16} \\
0, \text { otherwise }
\end{array}\right.
$$

For $0 \leq \mathrm{x} \leq 2,0 \leq \mathrm{y} \leq 1$. and:

$$
g(x, y)=\left(1+2 e^{-3\left(9 \sqrt{x^{2}+y^{2}}-6.7\right)^{-0.5}}\right),(x, y) \in(0,1) \times(0,1)
$$

The first dataset is taken from $f(x, y)$ consisting of 45 data points with 32 rectangles in rectangular domain. The second dataset is taken from $g(x, y)$ consisting of 25 data points with 16 rectangles. 
Similar to (Saaban et al., 2006; Kherd et al., 2014), the value of the free parameters $A, B$ and $C$ in Equation 19 are set to be $0.0003,2$ and 0.005 respectively which produced good quality of interpolating surfaces for all datasets. Figure $2 \mathrm{a}$ and Fig. $4 a$ show the quintic boundary curves in rectangular domain without imposing Proposition 1, while the boundary curves which satisfied the positivity preserving conditions are shown in Fig. $2 b$ and Fig. 4b for test functions $f$ and $g$ respectively. All the boundary curves in Fig. 2 b and Fig. 4 b are staying above the $X Y$-plane compared to the curves in Fig. 2 a and Fig. 4a where some parts of the curves lie below $X Y$-plane for both test functions.

The interpolating surfaces of non-positivity preserving quintic Said-Ball boundary curves for each test function are shown in Fig. 3a and Fig. 5a respectively where a large part of the surface crosses below the $X Y$-plane. Imposing on positivity preserving boundary curves reduses the numbers of point for the interpolating surfaces which crosses below the $X Y$-plane as shown in Fig. $3 b$ and Fig. 5 b.

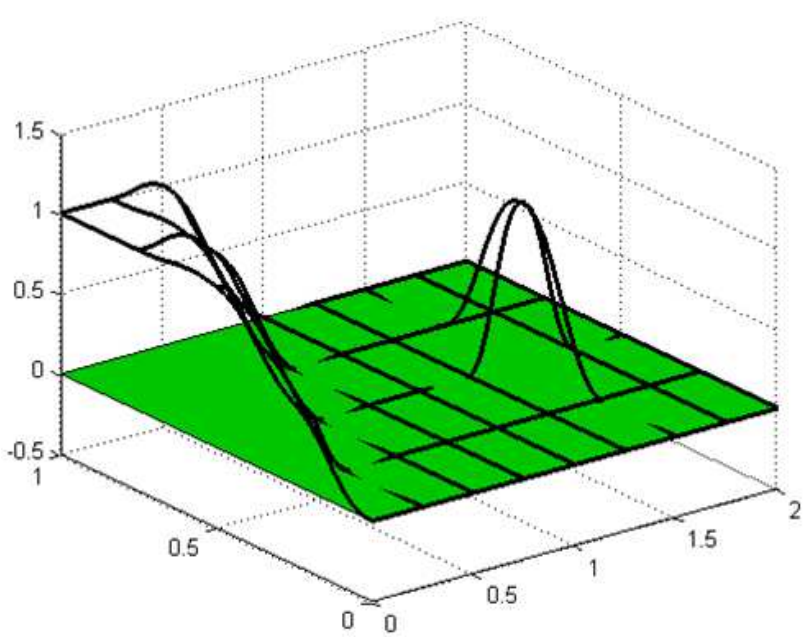

(a)

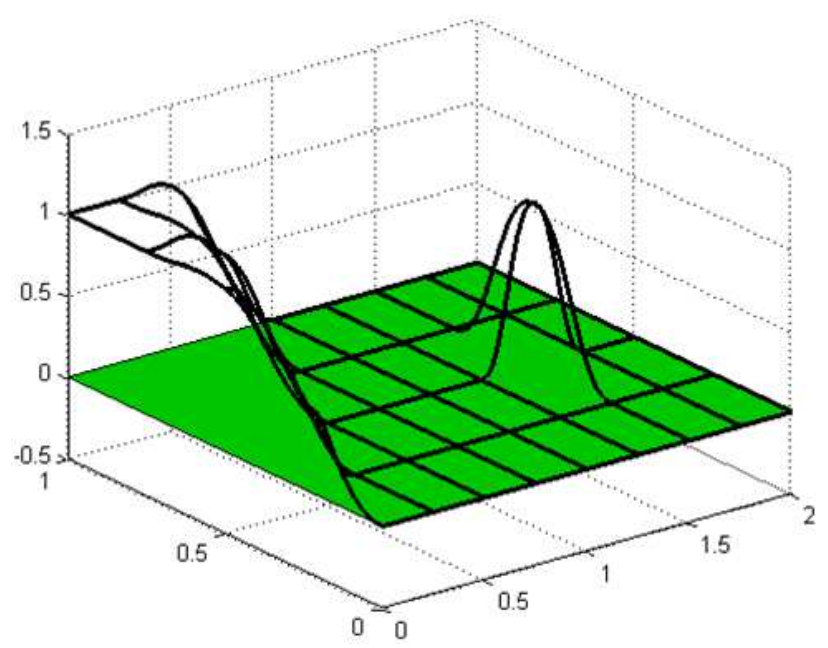

(b)

Fig. 2. Quintic Said-Ball Boundary curves (a) non-positivity preserving without imposing Proposition 1 (b) positivity preserving by imposing Proposition 1 (Test function $f(x, y)$

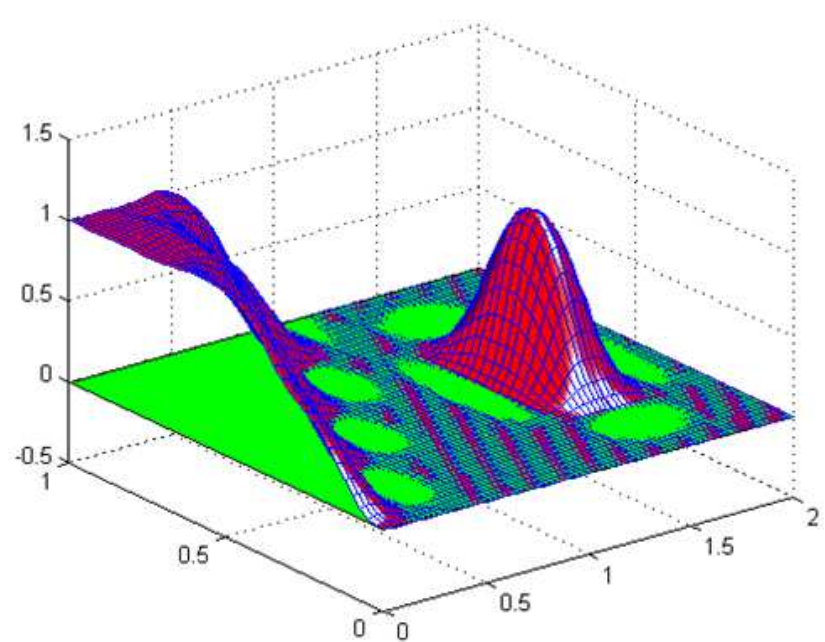

(a)

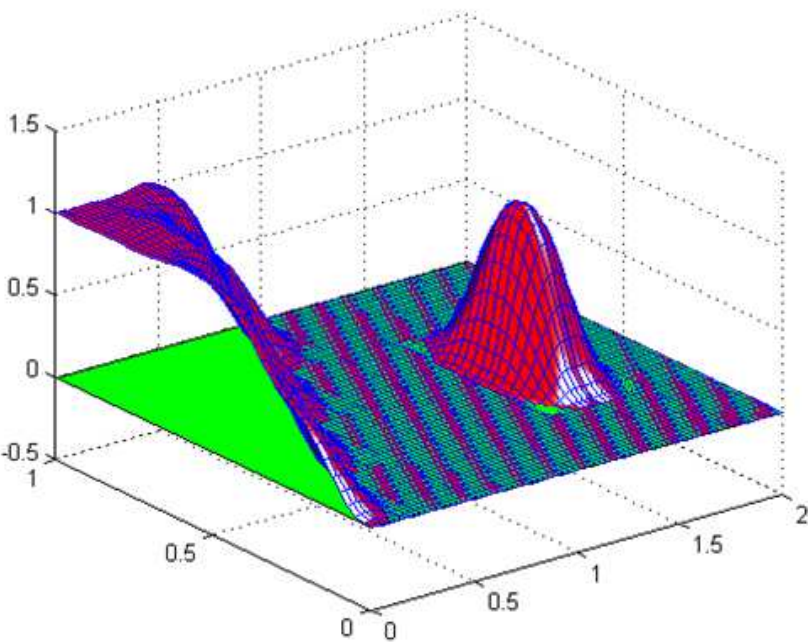

(b)

Fig. 3. Interpolating surfaces (a) using non-positivity preserving boundary curves (b) using positivity preserving boundary curves (Test function $f(x, y)$ 


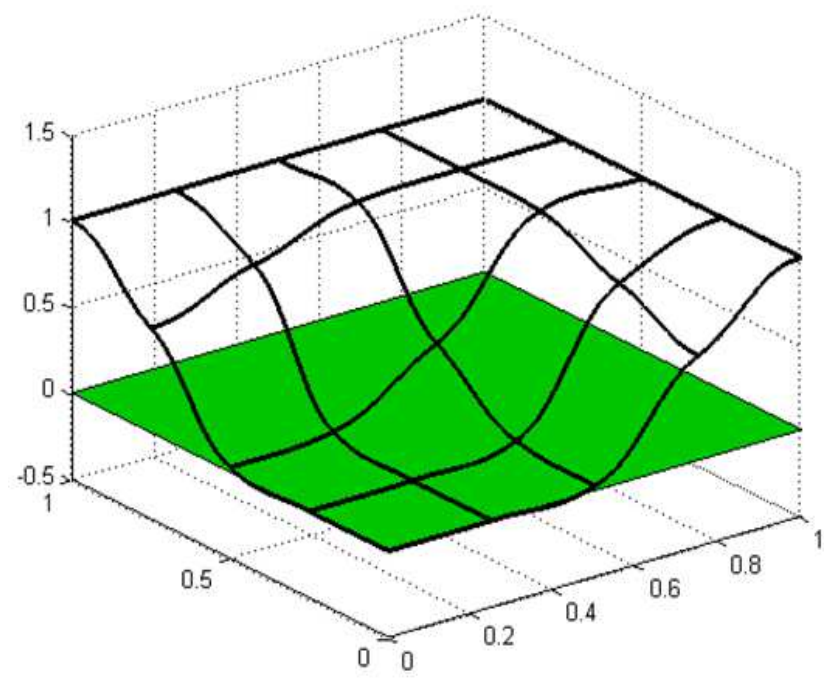

(a)

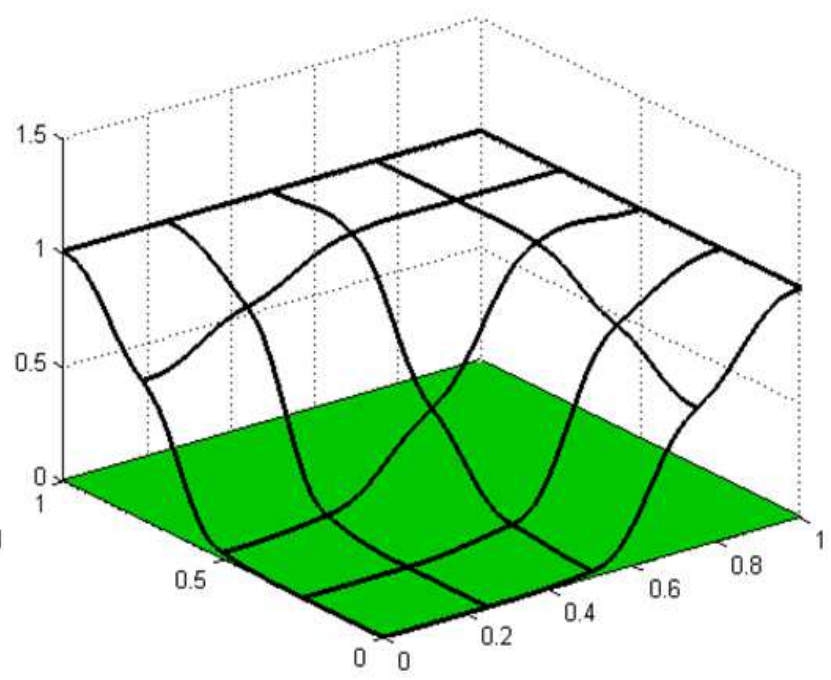

(b)

Fig. 4. Quintic said-ball boundary curves (a) non-positivity preserving without imposing proposition 1 (b) positivity preserving by imposing Proposition 1 (Test function $g(x, y)$ )

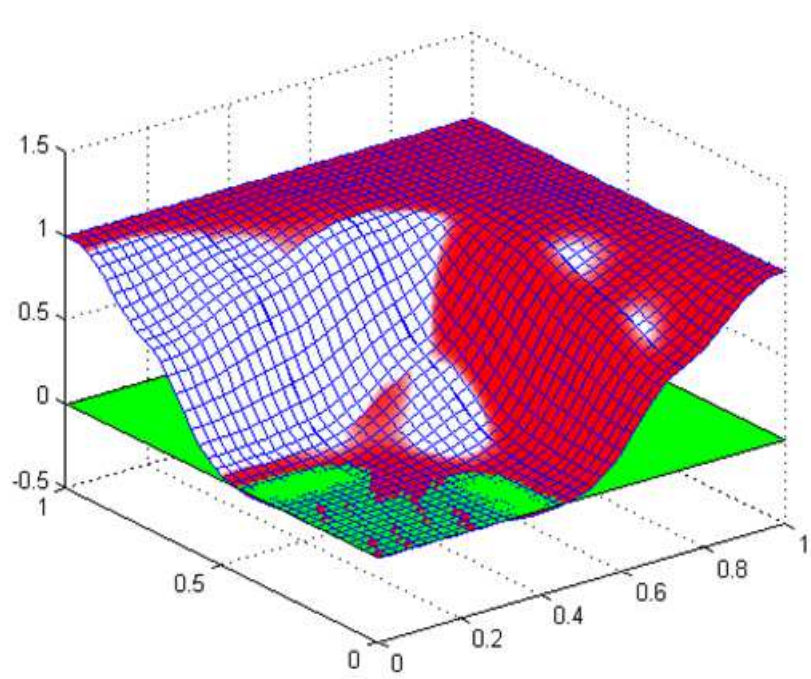

(a)

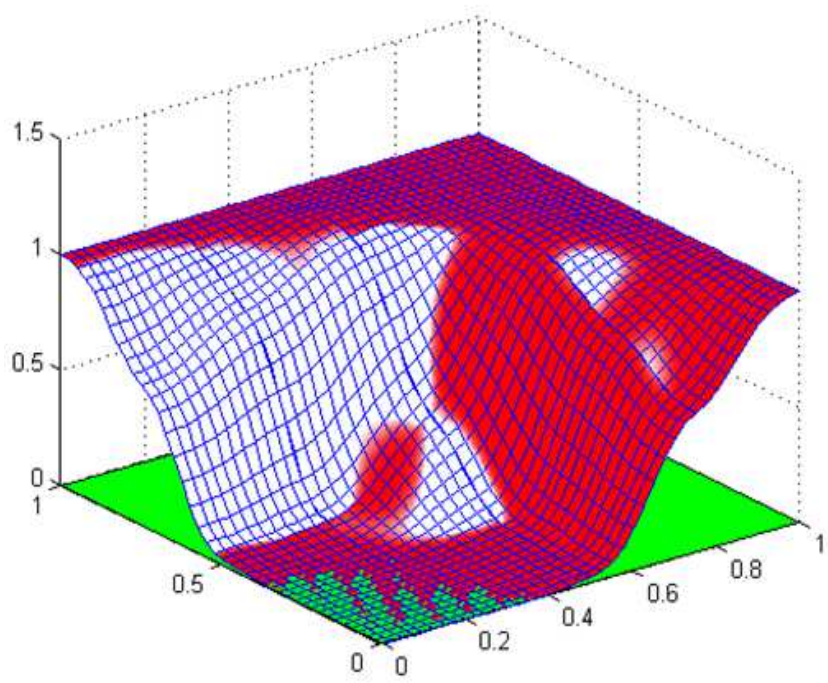

(b)

Fig. 5. Interpolating surfaces (a) using non-positivity preserving boundary curves (b) using positivity preserving boundary curves (Test function $g(x, y)$ )

\section{Conclusion}

In this study, a proposition is formulated of the sufficient condition for the positivity preservation of odd degree-n boundary curves. With the use of polynomial solution of fourth order linear elliptic PDEs, these curves are defined on a rectangular grid for the purpose of enhancing the positivity preservation of the interpolating surface. Through an independent adjustment of the lower boundary of edge ordinates, a sufficient condition on boundary curves for individual edge of degree $m$ by $n$ rectangular SaidBall patches is derived. After comparing between Said-Ball and Bézier as in Table 1 and 2, it was discovered that the result of Said-Ball is much better than of existing method for Bézier. The execution of the test functions indicates a well performance of the method proposed with regard to the preservation of the positivity of the boundary curves and also enhance the positivity preservation of the whole interpolating surfaces. 
Table 1. Comparison of the interpolating surfaces with non-positivity and positivity between Bézier and Said-Ball pre-serving boundary curves for the test function $f(x, y)$

\begin{tabular}{llllll}
\hline & & Number of points below XY-plane and percentage & Minimum value of function \\
Surface & $\begin{array}{l}\text { Number of } \\
\text { evaluation points }\end{array}$ & $\begin{array}{l}\text { Non-positivity preserving } \\
\text { boundary curves }\end{array}$ & $\begin{array}{l}\text { Positivity preserving } \\
\text { boundary curves }\end{array}$ & $\begin{array}{l}\text { Non- positivity } \\
\text { boundary curves }\end{array}$ & $\begin{array}{l}\text { Positivity preserving } \\
\text { boundary curves }\end{array}$ \\
\hline Bézier & 3321 & 1134 & 124 & -0.08232 & -0.022241 \\
& & $-34.15 \%$ & $-3.72 \%$ & -0.058653 & -0.02221 \\
Said-Ball & 3324 & 1148 & 104 & & \\
& & $34.54 \%$ & $3.13 \%$ & & \\
\hline
\end{tabular}

Table 2. Comparison of the interpolating surfaces between non-positivity and positivity between Bézier and Said-Ball preserving boundary curves for the test function $g(x, y)$

\begin{tabular}{llllll}
\hline & & Number of points below XY-plane and percentage & Minimum value of function \\
Surface & $\begin{array}{l}\text { Number of } \\
\text { evaluation points }\end{array}$ & $\begin{array}{l}\text { Non-positivity preserving } \\
\text { boundary curves }\end{array}$ & $\begin{array}{l}\text { Positivity preserving } \\
\text { boundary curves }\end{array}$ & $\begin{array}{l}\text { Non-positivity preserving } \\
\text { boundary curves }\end{array}$ & $\begin{array}{l}\text { Positivity preserving } \\
\text { boundary curves }\end{array}$ \\
\hline Bézier & 1681 & 286 & 6 & -0.039003 & -0.0004592 \\
& & $-17.01 \%$ & $-0.36 \%$ & -0.019917 & $2.31 \mathrm{E}-05$ \\
Said-Ball & 1681 & 246 & 0 & & \\
& & $14.63 \%$ & $0.00 \%$ & & \\
\hline
\end{tabular}

\section{Acknowledgment}

The authors are very grateful to the Ministry of Higher Education of Malaysia for providing us with the Fundamental Research Grant Scheme (FRGS) S/O number (12380) to enable us to pursue this research.

\section{Author's Contributions}

Ahmed Kherd: Participated in all experiments, coordinated the data-analysis and contributed to the writing of the manuscript and proofreading.

Azizan Saaban: Participated in all experiments, coordinated the data-analysis and contributed to the writing of the manuscript.

Noraziah Haji Man: Contributed to the writing of the manuscript and proofreading.

\section{Ethics}

This article is original and contains unpublished material. The corresponding author confirms that all of the other authors have read and approved the manuscript and no ethical issues involved.

\section{References}

Aphirukmatakun, C. and N. Dejdumrong, 2007. An approach to polynomial curve comparison in geometric object database. World Acad. Sci. Eng. Technol., 32: 161-167.

Arnal, A., J. Monterde and H. Ugail, 2011. Explicit polynomial solutions of fourth order linear elliptic partial differential equations for boundary based smooth surface generation. Comput. Aided Geometric Design, 28: 382-394.

DOI: $10.1016 /$ j.cagd.2011.06.001
Bloor, M.I. and M.J. Wilson, 1990. Representing pde surfaces in terms of $b$-splines. Comput. Aided Design, 22: 324-331.

DOI: $10.1016 / 0010-4485(90) 90083-O$

Conte, S.D. and S.D. de Boor, 1992. Elementary Numerical Analysis. 1st Edn., McGraw-Hil 1Kogakusha Ltd, Tokyo.

Hu, S.M, G.Z. Said and T.G. Jin, 1996. Properties of two types of generalized ball curves. Comput. Aided Design, 28: 125-133.

DOI: $10.1016 / 0010-4485(95) 00047-X$

Kherd, A., A. Saaban and N.H. Man, 2014. Surface interpolation using partial differentiation equation with positivity preserving cubic said-ball curves boundary condition. AIP Conf. Proc., 160, pp: 429-434. DOI: $10.1063 / 1.4882521$

Monterde, J. and H. Ugail, 2004. On harmonic and biharmonicbzier. Comput. Aided Geometric Design, 21: 697-715. DOI: 10.1016/j.cagd.2004.07.003

Monterde, J. and H. Ugail, 2006. A general 4th-order PDE method to generate Bézier surfaces from the boundary. Comput. Aided Geometric Design, 23: 208-225. DOI: 10.1016/j.cagd.2005.09.001

Saaban, A., A.R.M. Piah and A.A. Majid, 2006. Positivity-preserving scattered data interpolating surface using $C^{\wedge} 1$ piecewise cubic triangular patches. Proceedings of the International Conference on Computer Graphics, Imaging and Visualisation, Jul. 26-28, IEEE Xplore Press, Sydney, pp: 490-495. DOI: 10.1109/CGIV.2006.71

Saaban, A., A.R.M. Piah and A.A. Majid, 2007. Range restricted $C^{2}$ interpolant to scattered data. Proceedings of the Computer Graphics, Imaging and Visualisation, Aug. 14-17, IEEE Xplore Press, Bangkok, pp: 183-188. DOI: 10.1109/CGIV.2007.71 